\title{
Plasticized and nanofilled poly(lactic acid) nanocomposites: mechanical, thermal and morphology properties
}

\begin{abstract}
Poly(lactic acid) (PLA)-based nanocomposites filled with graphene nanoplatelets (xGnP) and containing epoxidized palm oil (EPO) as plasticizer were prepared by melt blending method. PLA was first plasticized by EPO to improve its flexibility and thereby overcome its problem of brittleness. Then, $\mathrm{xGnP}$ was incoporated into plasticized PLA to enhance its mechanical properteis. Plasticized and naonofilled PLA nanocomposites (PLA/EPO/xGnP) showed improvement in the elongation at break by $61 \%$ compared with plasticized PLA (PLA/EPO). The use of EPO and $\mathrm{xGnP}$ increases the mobility of the polymeric chains, thereby improving the flexibility and plastic deformation of PLA. The nanocomposites also resulted in an increase of up to $26.5 \%$ in the tensile strength compared with PLA/EPO blend. PLA/EPO reinforced with $\mathrm{xGnP}$ shows that increasing the $\mathrm{xGnP}$ content triggers a substantial increase in thermal stability. The TEM image of PLA/EPO/xGnP shows that the $\mathrm{xGnP}$ was uniformly dispersed in the PLA matrix and no obvious aggregation is observed.
\end{abstract}

Keyword: Graphene nanoplatelets; Plasticizer; Poly(lactic acid) 